\title{
Hacia un nuevo modelo de gestión del conocimiento caracterizado por la interacción de comunidades cognitivas*
}

Towards a New Knowledge Management Model Characterized by the Interaction of Cognitive Communities

\section{Para um novo modelo de gestão do conhecimento caracterizado pela interação de comunidades cognitivas}

\section{Amanda Vargas Prieto ${ }^{\star \star}$}

Fecha de recibido: 17 de mayo de 2014

Fecha de aprobado: 25 de noviembre de 2014

Doi: dx.doi.org/10.12804/rev.univ.empresa.28.2015.10

Para citar este artículo: Vargas Prieto, A. (2015). Hacia un nuevo modelo de gestión del conocimiento caracterizado por la interacción de comunidades cognitivas. Universidad \& Empresa, 17(28), 219-234. Doi: dx.doi. org/10.12804/rev.univ.empresa.28.2015.10

\footnotetext{
Esta investigación fue realizada gracias a una beca otorgada por la Universidad de Burdeos, la Federación Nacional de Cooperativas Agrícolas y el Instituto Nacional de Investigación Agronómica (INRA) en Francia. Fue realizada en el marco de una tesis de Doctorado en Ciencias Económicas de la Universidad de Burdeos, sustentada el 6 de septiembre de 2013.

** Doctora en Ciencias Económicas de la Universidad de Burdeos (Francia), Magíster en Inteligencia Económica de la Universidad de Angers (Francia), Administradora de Empresas del Politécnico Grancolombiano (Bogotá, Colombia). Profesora asociada de la Universidad de La Salle (Bogotá, Colombia) facultad de Ciencias Económicas y Sociales. Correo electrónico: amvargas@ unisalle.edu.co. Dirección postal: Universidad de La Salle sede Chapinero Cra. 5 No. 59A-44 Bogotá, Colombia.
} 


\section{RESUMEN}

La evolución del sector agrícola en Francia motivó la aparición de grupos empresariales formados por pequeñas cooperativas. Esto provocó una crisis de identidad de este tipo de empresas como consecuencia de la ruptura del vínculo entre sus miembros. Esta investigación, que utiliza métodos cualitativos, indaga en cinco grupos empresariales de cooperativas agrícolas francesas. Esto permite estudiar la interacción de los diferentes tipos de comunidades cognitivas por medio de la propuesta de un nuevo modelo de gestión del conocimiento caracterizado por la interacción de los grupos funcionales y las comunidades de práctica. Este modelo propuesto se denomina 'El modelo 3'.

Palabras clave: comunidades cognitivas, cooperativas agrarias, gestión del conocimiento, modelo 3, sector agrícola en Francia.

\section{ABSTRACT}

The evolution of the agricultural sector in France led to the appearance of entrepreneurial groups formed by small cooperatives. This caused an identity crisis among this type of companies due to a breakdown in the ties among its members. This research, which employed qualitative methods, studied five entrepreneurial groups of French agricultural cooperatives. This facilitated to study the interaction of different types of cognitive communities by proposing a new knowledge management model characterized by the interaction among functional groups and the communities of practice. The model proposed was named 'Model 3'.

Keywords: Cognitive communities, agricultural cooperatives, knowledge management, model 3, French agricultural sector.

\section{RESUMO}

A evolução do setor agrícola na França motivou a aparição de grupos empresariais formados por pequenas cooperativas. Isto provocou uma crise de identidade deste tipo de empresas como consequência da ruptura do vínculo entre seus membros. Esta pesquisa, que utiliza métodos qualitativos, indaga em cinco grupos empresariais de cooperativas agrícolas francesas. Isto permite estudar a interação dos diferentes tipos de comunidades cognitivas por meio da proposta de um novo modelo de gestão do conhecimento caracterizado pela interação dos grupos funcionais e as comunidades de prática. Este modelo proposto denomina-se 'O modelo 3'.

Palavras-chave: comunidades cognitivas, cooperativas agrárias, gestão do conhecimento, modelo 3, setor agrícola na França. 


\section{INTRODUCCIÓN Y MARCO TEÓRICO}

El sector de la cooperación agrícola en Francia se organizó principalmente bajo la influencia de tres grandes leyes (Confédération française de la coopération agricole [CFCA], 1999): la política agrícola de 1960 y de 1962, sobre la organización económica de los productores, y la ley de 1972 sobre el estatus jurídico de la cooperación agrícola. Tras el endurecimiento del entorno competitivo agrícola en Europa, relacionado con la globalización, se adoptan las leyes de 1991 y 1992. Esto se hizo con el fin de organizar las filiales y el fortalecimiento de los fondos propios de las cooperativas agrarias (Europe stratégie analyse financière [Eurostaf], 2007). Estas son responsables del desarrollo acelerado de los grupos cooperativos empresariales en la década del noventa (Forestier \& Mauget, 2000).

Estos grupos se han posicionado en el mercado agroalimentario europeo gracias a la combinación de cooperativas tradicionales y filiales o empresas privadas encargadas de la producción. La dualidad de los modos de gobernanza (asociativo y accionarial) de estos grupos cooperativos puede ser representada por un modelo híbrido que provoca una crisis de identidad en las cooperativas agrarias (Vargas Prieto, 2013). Esta crisis se traduce en varios efectos negativos relacionados con el surgimiento de grupos cooperativos en diferentes sectores de la economía.

En el sector agrícola, en particular, Forestier y Mauget (2001) describen tres efectos negativos de la creación de grupos cooperativos: 1) el distanciamiento, 2) la complejidad y 3) la confusión. Esta problematica en términos económicos se traduce como una pérdida de valor para los shareholders de las cooperativas. Teniendo en cuenta que el modelo de gobernanza híbrido específico de los grupos cooperativos reposa sobre una visión contractual de la firma, esta se ve limitada para entender el proceso de creación de valor en este tipo particular de organización (Vargas Prieto, 2013).

En efecto, el desarrollo de la actividad de asesoría a los agricultores es un lugar de creación y de difusión del conocimiento. La creación de valor en los grupos cooperativos está basada en la interaccion de asesores agrícolas y agricultores. Esta interacción es la fuente de las competencias de los grupos cooperativos y es el lugar en donde se puede crear conocimiento a través del intercambio del saber tácito de los agricultores y el saber codificado de los asesores. 
Conderando lo anterior, tenemos la necesidad de utilizar las teorias cognitivas de la firma para realizar nuestro análisis, pues estas otorgan una importancia considerable a la construcción de competencias y a las capacidades de innovación de la firma. En linea con el trabajo de Labarthe (2006), la asesoría agrícola es considerada como una actividad de servicio que permite la coproducción de conocimientos a través de la interacción de asesores y agricultores. Esta definición nos conduce a modificar el análisis clásico de la asignación de recursos en las organizaciones para poner en el centro de nuestras preocupaciones la producción del conocimiento.

El análisis de los marcos teóricos de los dos enfoques principales que constituyen la economía del conocimiento (la visión estratégica y la visión evolucionista), nos permite identificar sus principales limitaciones. Estos marcos teóricos no enfoncan su atención en los niveles medios de la organización, es decir, precisamente en los lugares donde los conflictos se resuelven y las creencias se negocian.

Según Cohendet y Diani (2003), estas teorías no profundizan en el análisis del proceso de construcción de conocimiento dentro de la organización. La visión estratégica adolece de contar con una metodología restringida (largo proceso y falta de herramientas de análisis) (Prahalad \& Hamel, 1990), al mismo tiempo se limita a explicar los procesos cognitivos centrándose en los administradores (Stalk, Evans, \& Shulman, 1992). Por otra parte, el enfoque evolucionista no tiene en cuenta los conflictos y las relaciones de poder dentro de la organización (Mangolte, 2000).

Los límites planteados justifican la elección de un enfoque basado en las comunidades cognitivas. Estas se interesan en la dinámica interna de la organización, es decir, en la creación del conocimiento en su interior. La lectura de la actividad de asesoría agricola en las cooperativas agrarias a través del prisma de las comunidades cognitivas nos permite entender el proceso de creación del conocimiento en este tipo de organizaciones. Las comunidades cognitivas son definidas por Muller (2004) como estructuras de interacciones sociales destinadas a la generación y difusión del conocimiento. Estas estructuras se caracterizan por ser lugares sociales de intercambio voluntario de experiencias y relaciones de confianza (Cappe, 2005; Diani, 2002).

En las comunidades cognitivas se crea y utiliza el conocimiento. Los miembros de la comunidad compar- 
ten una identidad (Vaast, 2003), un lenguaje (Foray, 2009), un acervo de conocimiento y una serie de intereses (Lewis, 1969). Existe además una proximidad cognitiva entre sus integrantes (Gallie \& Guichard, 2002).

Las comunidades cognitivas tienen tres características básicas, a saber: 1) una alta frecuencia de interacciones, 2) la presencia de un flujo de información identificada y 3) el acceso a la información por parte de cada uno de sus miembros (Bowles \& Gintis, 1998; Dupouët \& Tricot-Chamard, 2009; Dupouët, Yildizoglu \& Cohendet, 2003; Orr, 1996; Probst $\&$ Borzillo, 2007). Estos rasgos aseguran la creación de situaciones favorables a la difusión de la información y la adopción de procesos innovadores en las empresas.

Estas comunidades pueden adoptar la forma de asociaciones, sindicatos, federaciones, grupos de trabajo, etc. Los intercambios pueden tener lugar durante reuniones regulares o grupos de trabajo temáticos. Esto a través de materiales de información (periódicos, folletos) o, entre otras iniciativas, por medio de la organización de eventos (exposiciones, jornadas de puertas abiertas). También pueden utilizar las tecnologías de la información y las comunicaciones (TIC) para facilitar la interacción, incluso a través de foros, correo electrónico, listas de correo (Benghozi, Bitouzet, Soulier, \& Zacklad, 2001). Además de las comunidades entre empresas, la literatura también se refiere a múltiples ejemplos de comunidades dentro de la propia empresa (Brown \& Duguid, 1998).

\subsection{La organización concebida como una comunidad de comunidades}

Los criterios utilizados para distinguir las comunidades dentro de una empresa pueden ser las formas en que aprenden y participan en el desarrollo y la expansión del conocimiento organizacional (Cohendet, Créplet, \& Dupouët, 2003), sus objetivos, las características de sus miembros, su actividad cognitiva dominante, sus criterios de selección de personal, su modo de producción de conocimiento o su estilo de aprendizaje (Cohendet, Roberts, \& Simon, 2010). Pueden ser determinados también en función de su orientación. Algunos de estos están más orientados hacia la creación de conocimiento, mientras otros lo están a la acción. Unos están definidos y controlados por mecanismos de información específicos, otros son más independientes (Creplet, 2001; Creplet, Dupouët, Kern \& Munier, 2001).

Estas características permiten a Cohendet, Créplet y Dupouët (2003) 
establecer una clara distinción entre las llamadas comunidades jerárquicas y las comunidades autónomas. Las comunidades jerárquicas pueden ser de tres tipos: 1) las redes, 2) los grupos funcionales y 3) los equipos. Están dirigidas por las instancias jerárquicas dentro de la empresa. En este tipo de comunidad el objetivo es la difusión del conocimiento y, si existe creación de conocimiento, esto se da de manera involuntaria.

Las comunidades autónomas, por su parte, reposan sobre la adhesión de los miembros a una pasión compartida o a un conjunto de reglas llamado 'autoridad procesal' (Cohendet, Créplet, \& Dupouët, 2003). La literatura identifica dos tipos de comunidades autónomas: 1) las comunidades epistémicas y 2) las comunidades de práctica. Las primeras están orientadas hacia la creación de nuevo conocimiento (Cohendet \& Diani, 2003), las segundas están creadas para lograr una actividad productiva en la que la generación de conocimiento se realice de manera voluntaria (Brown \& Duguid, 1991; Lave \& Wenger, 1991; Orr, 1996).
1.2. La interacción de las comunidades para la gestión del conocimiento dentro de la empresa

Según Hatchuel (1999), para comprender el proceso de creación de conocimiento en la empresa, como un sistema de acción colectiva, es necesario estudiar su interacción. Según Cohendet, Créplet y Dupouët (2003) y Cohendet y Diani (2003), la gestión del conocimiento en las organizaciones se encuentra en parte en la interacción entre dos comunidades jerárquicas: por un lado, los grupos funcionales y los equipos de trabajo (modelo 1) y, por el otro, en la interacción entre las dos comunidades autónomas: comunidades de práctica y las comunidades epistémicas (modelo 2).

Sin embargo, Vargas Prieto (2013) demuestra que estos dos modelos no son suficientes para explicar la creación de conocimiento en los grupos cooperativos agrarios. Este autor señala que la organización de la actividad de asesoramiento agrícola en los grupos cooperativos puede ser representada por las comunidades jerárquicas (grupos funcionales) y

1 Autoridad procesal puede definrse como "un conjunto de reglas o códigos de conducta que define los objetivos de la comunidad y la forma de regular la conducta colectiva dentro de la comunidad para el logro de los objetivos" (Cohendet \& Diani, 2003, p. 705). 
las comunidades autónomas (comunidades de práctica).

En efecto, la actividad de asesoramiento en los grupos cooperativos se realiza a través de tres prácticas asociativas (Coté, 2007): la información, la formación y la consultación. Este último es específico de las empresas de la economía social y solidaria y permite a la organización la creación de canales de comunicación entre la organización y los diferentes shareholders. Esta especificidad de los grupos cooperativos agrícolas no puede ser analizada por los dos modelos de gestión del conocimiento anteriormente descritos, puesto que no tienen en cuenta la interacción de las comunidades de distinta naturaleza. Sin embargo, la práctica de la consulta se lleva a cabo en la interacción entre el grupo funcional y la comunidad de práctica.

\subsection{La forma y la frecuencia de} los intercambios, las principales características del tercer modelo de interacción

En el 'modelo 3', que proponemos aquí, la gestión del conocimiento reside en la interacción entre los grupos funcionales y las comunidades de práctica. Esta interacción se lleva a cabo por los intercambios entre los asesores del back-office y los del front-office, por una parte, y entre los miembros y los asesores, por la otra (ver figura 1).

Figura 1. Creación y difusión del conocimiento en los grupos cooperativos agrarios

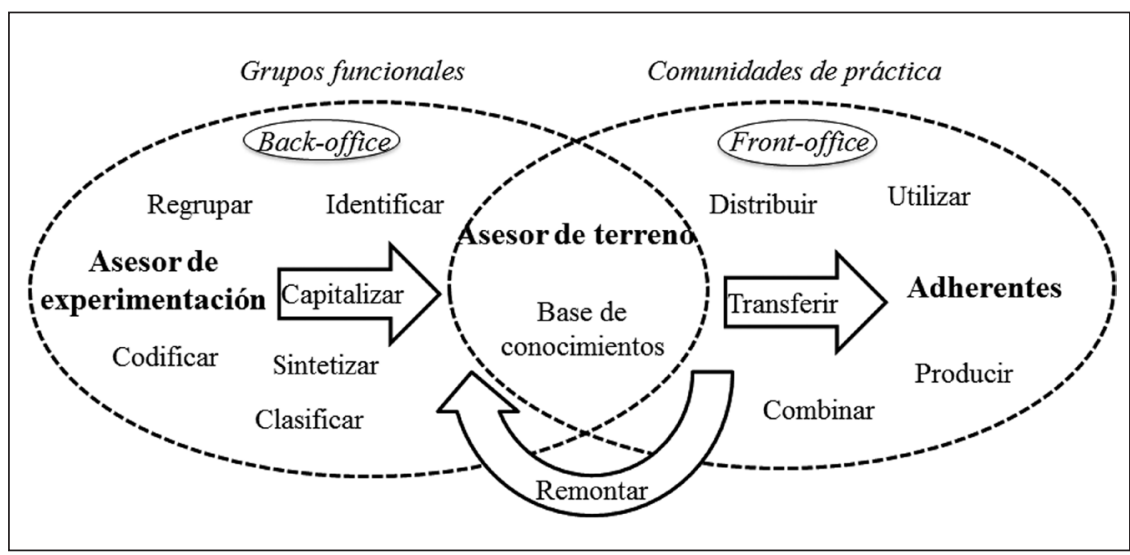

Fuente: elaboración propia.

En el modelo 3 el proceso de creación de conocimiento se lleva a cabo en espiral. Esto implica que el conocimiento tácito de los agricultores se 
codifica en los grupos funcionales, $\mathrm{y}$ el conocimiento codificado de los asesores de los grupos funcionales se utiliza para crear nuevo conocimiento tácito. De hecho, el nuevo conocimiento codificado (boletines, documentos informativos, etc.) dentro de los grupos funcionales se distribuye nuevamente a los miembros. A través de este doble proceso de top down, se muestra cómo en los grupos cooperativos agrarios se desarrollan nuevos conocimientos.

\subsection{La función principal del asesor en el tercer modo de interacción}

El papel del asesor agrícola en la creación y difusión del conocimiento en grupos cooperativos agrarios se destaca. Comenzamos definiendo el asesor como un intermediario entre los grupos funcionales y comunidades de práctica en los grupos cooperativos. Esta definición se ha adaptado al nuevo contexto agrícola. Inicialmente el asesor técnico era visto principalmente como un actor que transmitía a los agricultores los conocimientos y las competencias certificadas por la ciencia.

Según Mundler, Labarthe y Laurent (2006), el conocimiento en agricultura se difunde y es fácilmente creado gracias a las capacidades de anticipación, de análisis, de escucha y de reformulación de los problemas de los asesores, así como a su saber en materia de análisis económico y financiero y, evidentemente, a su dominio de los demás conocimientos técnicos relevantes. La literatura demuestra que los asesores tienen cierto grado de autonomía en la creación de la asesoría (Brives,2001). De hecho, estos ajustan los conocimientos basados en su experiencia antes de transmitirlos a los agricultores. Esto puede ser visto como un proceso de traducción, cuando los asesores tienen un papel de 'traductores' en el sentido de Callon (2003). De este modo, los asesores pueden ser considerados actores 'multiposicionados', que traducen lenguajes y preocupaciones de cada comunidad (Brown \& Duguid, 1991).

A través de la noción de traducción, propuesta por Callon y Latour (1990), podemos entender la arquitectura cognitiva de los grupos cooperativos agrícolas. Es entonces a través de la traducción que estas agrupaciones codifican sus experiencias y la historia de la empresa para dar sentido a la identidad organizacional mediante la creación de una visión o identidad compartida.

Las comunidades sin intermediarios permanecen aisladas y dispersas, sin ninguna identidad (Callon et al., 1999). Es así que el intercambio entre los grupos funcionales y las comunidades de práctica al interior 
de los grupos cooperativos agrarios permiten reforzar el vínculo con los asociados, desarrollando de esta manera la identidad organizacional. Cohendet y Diani (2003) proponen analizar las interacciones entre las diversas comunidades dentro de la empresa a partir de dos mecanismos principales:

- La repetitividad de las interacciones entre las comunidades: es el criterio cuantitativo de la comunicación entre las comunidades. Permite conocer la frecuencia de las reuniones así como la creación de condiciones favorables de resolución de conflictos (Cohendet \& Diani, 2003, p. 709).

- La naturaleza de la comunicación entre las comunidades: es un lenguaje común que facilita la comunicación y la creación del conocimiento. Esto se observa, por ejemplo, en el caso de comunidades como las involucradas en situaciones de emergencia (socorristas, bomberos, etc.), en donde el número de interacciones es bajo, pero los actores se pueden coordinar de inmediato gracias al conocimiento de un lenguaje común y de normas conocidas y compartidas por todos (Cohendet \& Diani, 2003).

En resumen, el uso simultáneo de los dos mecanismos descritos anteriormente facilita el análisis de la calidad de la interacción entre las dos comunidades, de diferente naturaleza, identificadas al interior de los grupos cooperativos agrícolas. De hecho, estas dos comunidades cognitivas pueden tener muchas interacciones y no entenderse o tener todos los medios cognitivos para comprenderse y, al final, no interactuar de manera frecuente.

\section{MÉTODOS}

A continuación, presentamos brevemente el marco metodológico de nuestra investigación. Comenzamos justificando la elección del carácter exploratorio y cualitativo para comprender la organización de la asesoría agrícola: seleccionamos la exploración cualitativa porque los conceptos que se estudian no están suficientemente definidos en la literatura. Este es el caso del asesoramiento agrícola en los grupos cooperativos franceses. Elegimos la metodología del estudio de caso, debido a que este se encuentra destinado para la descripción a profundidad y análisis de una pequeña muestra.

Yin (1990) propone una definición de esta herramienta. Según este autor:

Un estudio de caso es una investigación empírica que examina un fenómeno contemporáneo dentro de su contexto de vida real cuando los 
límites entre el fenómeno y el contexto no son claramente evidentes y en el que múltiples fuentes de datos son utilizados. (p. 17)

De hecho, Yin (2009) afirma que el comportamiento de un grupo y los procesos organizacionales deben ser estudiados a través del estudio de caso. Según Albarello (2011), este tipo de estudio es particularmente apropiado en la investigación de ciertas situaciones, especialmente cuando se trata de analizar actividades, programas o grupos o cuando el fenómeno estudiado está inextricablemente ligado al contexto en el que apareció y se desarrolló.

Según Creswell (2007) y Yin (2009), este tipo de investigación es muy eficaz cuando se trata de probar hipótesis que implican la comprensión de una situación global o para identificar las características específicas de un evento, actividad o programa. Lo elegimos entonces, ya que este permite la identificación de las características de la actividad de asesoría agrícola de los grupos cooperativos.

Por otro lado, el uso de múltiples fuentes de datos nos permite exami- nar las diferentes estratégias adoptadas por los grupos de cooperación para enfrentar la crisis de identidad. También podemos determinar si las comunidades cognitivas de diferente naturaleza en los grupos cooperativos agrícolas interactúan a través de la intervención de los asesores agrícolas. Por lo tanto, elegimos una estratégia de investigación basada en el estudio de casos seleccionados bajo criterios de representación teórica, variedad, equilibrio y potencial para el descubrimiento de nuevos objetos. Con base en lo anterior, seleccionamos cinco grupos cooperativos (GC) agrícolas que son representativos de los diferentes grupos cooperativos agrícolas existentes en Francia.

Por último, detallamos ahora la técnica utilizada para la producción de datos. Esta se compone de dos fases: 1) una fase exploratoria inicial, que facilitó la creación de una lista de observación, y 2) una segunda fase que incluyó la realización de entrevistas semiestructuradas, acompañadas de la observación directa y del estudio de los documentos internos de cada grupo. Nuestroenfoquesepresentade manera sintética y gráfica en la figura 2. 
Figura 2. Estructura de la investigación

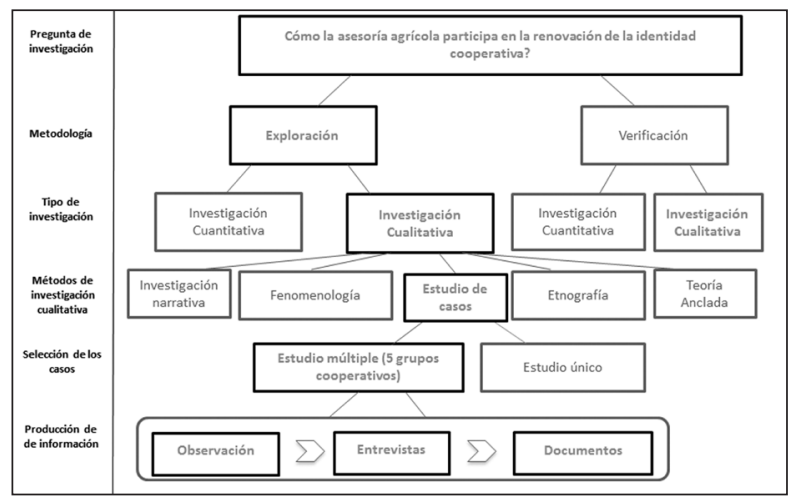

Fuente: elaboración propia.

\section{RESULTADOS}

Hemos estudiado previamente dos mecanismos que promueven el vínculo entre la cooperativa y los asociados: el nivel de intervención de los asesores (frecuencia de interacciones) y el nivel de desarrollo de la estrategia de la organización (formas de intercambio). De hecho, hemos puesto en evidencia que el flujo de conocimiento entre las comunidades de diferentes tipos mejora la identidad cooperativa.
Esta identidad es entonces el conjunto de rutinas, normas, convenciones y conocimiento común que guían el comportamiento colectivo de la organización. Dado que la identidad de los grupos cooperativos depende tanto de lo uno como de lo otro, cruzamoslos datosquetenemos sobre estos dos elementos para llegar a conclusiones respecto a la crisis de identidad en cada grupo de estudio. La tabla 1 resume los resultados obtenidos.

Tabla 1. Resultados de los grupos estudiados

\begin{tabular}{|l|c|c|}
\hline \multicolumn{1}{|c|}{ Nivel } & $\begin{array}{c}\text { Nivel de intervención } \\
\text { de los asesores }\end{array}$ & $\begin{array}{c}\text { Nivel de desarrollo de la estratégia } \\
\text { de la asesoría }\end{array}$ \\
\hline Elevado & GC*3 & GC4 \\
\hline Relativamente elevado & GC4 & GC1 \\
\hline Intermedio & GC5 & GC5 \\
\hline Moderado & GC1 & GC3 \\
\hline Bajo & GC2 & GC2 \\
\hline
\end{tabular}

* Grupo cooperativo (GC) agricola

Fuente: elaboración propia. 
Tres tendencias resultan del análisis de la tabla 1:

- El buen posicionamiento de GC4 en los dos indicadores, lo que parece ser favorable a la interacción de los grupos funcionales y las comunidades de práctica.

- La posicion intermediaria de GC5, que se ubica en el medio en los dos indicadores con respecto a los otros grupos cooperativos. En ese contexto, la interacción de las comunidades cognitivas permite todavía la creación de conocimiento.

- El mal posicionamiento de GC2 en los dos indicadores.

Proponemos entonces una representacion gráfica en forma de ejes, en donde posicionamos a los cinco grupos cooperativos agrícolas estudiados, ubicando el nivel de intervención de los asesores en las abscisas y el nivel de desarrollo de la estrategía de asesoría en las ordenadas (ver figura 3).

Figura 3. Identidad cooperativa de los grupos estudiados

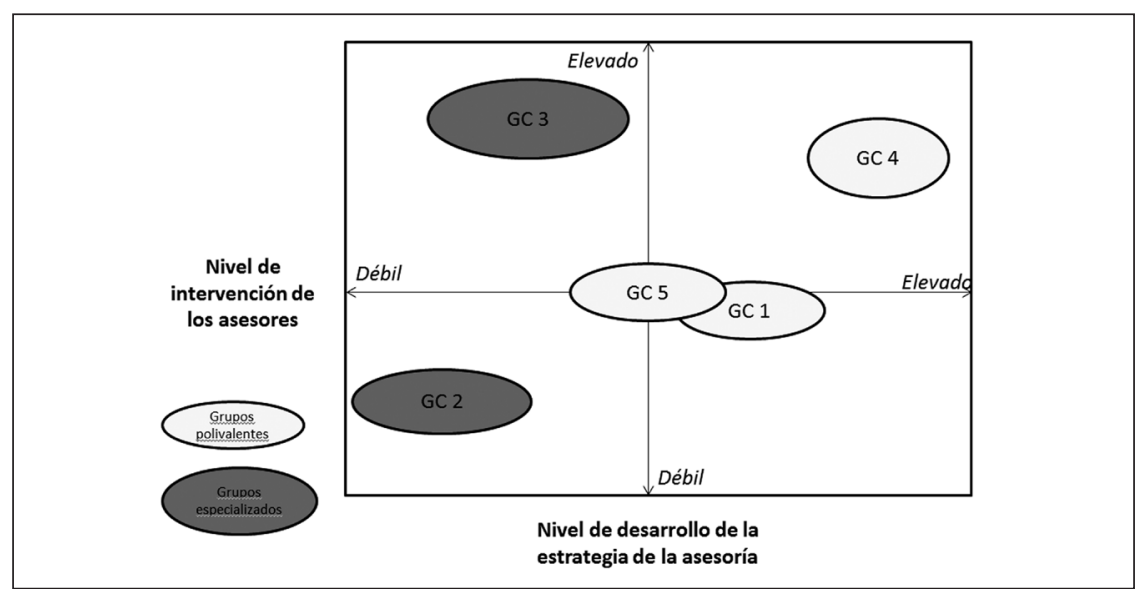

Fuente: elaboración propia.

Finalmente, se considera que los criterios de selección que nos hemos fijado para nuestra muestra son relevantes. Esto en la medida en que logramos identificar todos los tipos de identidad posibles en nuestros resultados, lo cual también nos ha permi- tido identificar un nuevo caso no especificado en la literatura: grupo con un nivel de identidad intermedio.

Por otra parte, en la figura 3 se pueden identificar tres grupos que se diferencian de los demás: GC4, que tie- 
ne los dos niveles altos; GC5, que está en el medio del eje, y GC2, que tiene dos niveles bajos.

Ahora bien, si se realiza un análisis con respecto al tipo de producción de cada grupo (polivalentes/especializados), es posible encontrardiferencias. Por un lado, los grupos especializados (en rojo en la figura) se encuentran en un nivel de desarrollo de estrategia limitado (bastante bajo). Por otro lado, los grupos polivalentes no tienen un alto nivel de desarrollo de la estrategía y el nivel de la frecuencia de intervención es bastante variable. De hecho, los tres grupos polivalentes se encuentran en los tres grados del eje (alta para GC4, media para GC5 y baja para GC1).

\section{DISCUSIÓN Y CONCLUSIONES}

Nuestros resultados nos llevan a reconocer la contribución de la asesoría agrícola en la resolución de la crisis de identidad de los grupos cooperativos agrícolas franceses. $\mathrm{Al}$ movilizar las comunidades cognitivas para comprender la gestión del conocimiento en la organización de los grupos cooperativos agrarios, se destaca la existencia de grupos funcionales y comunidades de práctica en este tipo de organización.

La combinación de estas dos comunidades permite avanzar hacia la proposición de un nuevo modelo de gestión del conocimiento: el modelo 3 . En este modelo se destaca el papel de los asesores agrícolas. Nuestra contribución teórica es original en tanto pocos estudios han examinado la relación entre las comunidades de diferentes tipos al interior de una misma organización.

Por último, el cruce de dos indicadores (frecuencia de la interacción y formas de intercambio) nos permitió analizar el flujo de conocimiento entre las dos comunidades de diferente naturaleza y evaluar la organización de la actividad de asesoría agrícola en la resolución de la crisis de identidad en cada grupo de estudio. Hemos identificado también varias situaciones posibles de identidad cooperativa y destacado la existencia de un nuevo caso no especificado en la literatura.

\section{REFERENCIAS}

Albarello, L. (2011). Choisir l'étude de cas comme méthode de recherche. Bruxelles - París: De Boeck. Benghozi, P. J., Bitouzet, C., Soulier, E., \& Zacklad, M. (2001). Le mode communautaire: vers une nouvelle forme d'organisation. Ponencia presentada al $3 \mathrm{e}$ Colloque International sur les Usages et Services des Télécommunications: EUSAGES, France 
Telecom R\&D, ENSTParis, IREST, ADERA, París, Francia.

Bowles, S., \& Gintis, H. (1998). The moral economy of communities: Structured populations and the evolution of prosocial norms. Evolution \& Human Behavior, 19(1), 3-25.

Brives, H. (2001). Mettre en technique. Conseillers agricoles et pollution de l'eau en Bretagne, Ruralia.

Brown, J. S., \& Duguid, P. (1998). Organizing knowledge. California Management Review, 40(3), 90-111.

Brown, J., Duguid, P. (1991). Organizational learning and communities of practice: Toward a unified view of working, learning and innovation. Organization Science, 2(1), 40-57.

Callon, M. (2003). The increasing involvement of concerned groups in R\&D policies: What lessons for public powers? En A. Geuna, A. Salter \& W. E. Steinmueller (Eds.), Science and innovation: Rethinking the rationales for funding and governance (pp. 3068). Cheltenham: Edward Elgar. Callon, M., \& Latour, B. (1990). La science telle qu'elle se fait. París: La Découverte.

Callon, M., Cohendet, P., Curien, N., Dalle, J. M., Eymard Duvernay, F., Foray, D., \& Schenk, E. (1999). Réseau et coordination. París: Economica.
Cappe, E. (2005). Identification de graines de communauté de pratique: mise en œuvre au sein d'une entreprise de microélectronique. Ponencia presentada a la XIVème Conference de l'Association International de Management Stratégique (AIMS). Angers, Francia.

Cohendet, P., \& Diani, M. (2003). L'organisation comme une communauté de communautés: croyances collectives et culture d'entreprise. Revue d'Économie Politique, 113(5), 697-721.

Cohendet, P., Créplet, F., \& Dupouët, O. (2003). Innovation organisationnelle, communautés de pratique et communautés épistémiques: le cas de Linux. RevueFrançaise de Gestion, 146, 99-121.

Cohendet, P., Roberts, J., \& Simon, L. (2010). Créer, implanter et gérer des communautés de pratique. Gestion, 35(4), 31-35.

Confédération française de la coopération agricole (CFCA) (1999). L'évolution du secteur coopératif agricole [document de travail]. París: CFCA

Côté, D. (2007). Fondements d'un nouveau paradigme coopératif (NPC): quels incitatifs pour les acteurs clés? Working paper, HEC Montréal, janvier.

Créplet, F. (2001). Pour une Approche des PME: Leur Evolution et Leur Développement dans une 
Perspective Cognitive - Entre Communautés d'Action et Communautés de Savoir, Thèse de Doctorat, Bureau d'Economie Théorique et Appliquée, Strasbourg.

Créplet, F., Dupouët, O., Kern, F., \& Munier, F. (2001). Dualité Cognitive et Organisationnelle de l'Entreprise: le Rôle Différencié du Manager et de l'Entrepreneur, Revue d'économie industrielle, 95, pp. 9-22.

Creswell, J. (2007). Qualitative inquiry and research design: Choosing among five approaches. London: SAGE.

Diani,M.(2002).Connaissanceetperformance économique: Une nouvelle vision de la firme dans une économie basée sur la connaissance, Communication au Colloque Interdisciplinaire «Connaissance(s) et Incertitude» Aix-enProvence 27 septembre 2002, pp. 20.

Dupouët, O., \& Tricot Chamard, I. (2009). Membre d'une communauté de savoir et salarié d'une firme: enjeux et perspectives en droitdu travail. En B. Baudry \& B. Dubrion(Eds.),Analyses ettransformations de la firme (pp. 253275). París: La Découverte.

Dupouët, O., Yildizoglu, M., \& Cohendet, P. (2003). Morphogenèse de communautés de pratique. Revue d'Économie Industrielle, 103(la morphogénèse des réseaux), 91-110.

Europe stratégie analyse financière (Eurostaf) (2007). Les mutations et les perspectives de développement des coopératives agricoles en france. Paris: Eurostaf.

Foray, D. (2009). L'économie de la connaissance. París: La Découverte.

Forestier, M., \& Mauget, R. (2000). De la coopérative au groupe coopératif agro-alimentaire, $1^{\text {èr }}$ partie: de la valeur pour l'usager à la valeur pour l'actionnaire?, REC$M A$ - Revue Internationale de L'Économie Sociale, 278, 1-15.

Forestier, M., \& Mauget, R. (2001). De la coopérative au groupe agro-alimentaire. RECMA - Revue Internationale de L'Économie Sociale, 279, 60-70.

Gallié, E.P., \& Guichard, R. (2002). The impact of ICT sophistication on geographically distant networks: the case of space physics as seen from France, NPR Net Conference, March 21-23, Brighton.

Hatchuel, A. (1999). Connaissances, modèles d'interaction et rationalisations - De la théorie de l'entreprise à l'économie de la connaissance. Revue d'Économie Industrielle, 88(1), 187-209. Labarthe, P. (2006). La privatisation $d u$ conseil agricole en question. Evolutions institutionnelles et 
performances des services de conseil dans trois pays européens (Allemagne, France, Pays-Bas). Doctorado en Ciencias Económicas, tésis de doctorado inédita, Universidad de París XII, París, Francia.

Lave, J., \& Wenger, E. (1991). Situated learning: Legitimate peripheral participation. Cambridge - Nueva York: Cambridge University Press.

Lewis, D. K. (1969). Convention: A philosophical study. Cambridge: Harvard University Press.

Mangolte, P. A. (2000). Organisational learning and the organisational link: The problem of conflict, political equilibrium and truce. European Journal of Economic and Social Systems, 14(2), 173-190.

Muller, P. (2004). Coordination des communautés de pratique: les rôles différenciés de la réputation et de la confiance. Tesis de Doctorado en Ciencias Económicas, tesis de doctorado inédita, Universidad Louis Pasteur - Estrasburgo I, Francia.

Mundler, P., Labarthe, P., \& Laurent, C. (2006). Les disparités d'accès au conseil agricole: le cas de la région Rhône-Alpes. Economie Rurale, 291, 26-41.
Orr, J. E. (1996). Talking about machines: An ethnography of a modern job. Ithaca: ILR Press.

Prahalad, C. K., \& Hamel, G. (1990). The core competence of the corporation. Harvard Business Review, 68(3), 79-91.

Probst, G., \& Borzillo, S. (2007). Piloter les communautés depratique avec succès. Revue Française de Gestion, 1(170), 135-153.

Stalk, G., Evans, P. \& Shulman, L. (1992). Competing on capabilities: The new rules of corporate strategy, Harvard Business Review, 70(2), 57-69.

Vaast, E. (2003). Les communautés de pratique sont elles pertinentes? Ponencia presentada a la XIème Conférence Annuelle de l'Association International de Management Stratégique(AIMS), Paris, Francia.

Vargas Prieto, A. (2013). Le rôle du conseil agricole dans la gouvernance coopérative. Doctorado en CienciasEconomicas, tesisdedoctoradoinédita,UniversidadMontesquieu - Burdeos IV, Francia.

Yin, R. K. (1990). Case study research: Design and methods (2nd ed.). Beverly Hills: SAGE.

Yin, R. K. (2009). Case study research: Design and methods (4th ed.). Londres: Sage. 